\title{
ERNST JÜNGER Y EL INSTANTE DE CRISIS Humberto Beck*
}

\section{ERNST JÜNGER AND THE MOMENT OF CRISIS}

RESUMEN: La noción de instante (el momento presente, el ahora) es una de las principales categorías para analizar la experiencia del tiempo en la modernidad. Por su vínculo con el fenómeno de "lo repentino", el instante se revela como un concepto privilegiado para explorar las experiencias de crisis y ruptura histórica. El autor alemán Ernst Jünger (1895-1998) ofrece en su producción literaria de los años de la República de Weimar — las memorias de su experiencia en la Primera Guerra Mundial, sus escritos literarios y sus ensayos de análisis político y cultural - una las encarnaciones más reveladoras de la noción de temporalidad instantánea. En la obra de Jünger, el concepto de instante arroja luz, en particular, sobre el fenómeno de la transformación de la experiencia y la percepción en las nuevas condiciones de la sociedad urbana e industrial.

PALABRAS ClaVE: Ernst Jünger, Weimar, Primera Guerra Mundial, temporalidad, instante, lo repentino, crisis, percepción, experiencia
ABSTRACT: The notion of instant (the present moment, now) is one of the main categories for the analysis of the experience of time in Modernity. Due to its connection to the phenomenon of "suddenness", the instant is seen as a useful concept to examine the experiences of historical crises and ruptures. The German author, Ernst Jünger (1895-1998), offers us in his literary work in the Weimar Republic years - memoirs of his experience in World War I, his literary compositions and his essays of political and cultural analysis - one of the most revealing incarnations of the concept of temporality of the instant. In his work, the concept of the instant sheds light especially on the phenomenon of the transformation of the experience and the perception of new conditions in the industrial and urban society.

KEYWORDS: Ernst Jünger, Weimar, World War I, temporality, instant, suddenness, crisis, perception, experience.

* Departamento de Historia, Universidad de Princeton. 


\section{ERNST JÜNGER Y EL INSTANTE DE CRISIS}

En este artículo se examina el concepto del instante (el momento presente, el ahora) como la categoría temporal para la experiencia de la crisis y la ruptura súbita en la obra del escritor alemán Ernst Jünger. En mi opinión, la noción de instante es un elemento teórico decisivo para comprender la naturaleza de la conciencia histórica durante las primeras décadas del siglo XX en Europa en general, y en particular en Alemania durante los años de la Primera Guerra Mundial y la República de Weimar. Quiero mostrar que esta afirmación se sostiene sobre la existencia de una afinidad conceptual entre la categoría temporal del instante y las nociones de modernidad y de crisis. Asimismo, argumentaré que los escritos de entre- guerras de Jünger (su obra autobiográfica y literaria sobre la guerra y sus ensayos de política sobre la naturaleza de la sociedad de masas tecnológica) ejemplifican la correspondencia entre instante y crisis de una manera especialmente atractiva, pues representa la crisis de la experiencia como la contraparte subjetiva e individual de la sensación de ruptura histórica de ese periodo.

\section{Ernst Jünger}

Ernst Jünger (1895-1998) fue uno de los más importantes y controvertidos escritores alemanes del siglo XX. La prolífica carrera literaria de Jünger comenzó en 1920 con la publicación 
de Tempestades de acero, memorial de sus experiencias como oficial de tropas de choque del Ejército alemán durante la Primera Guerra Mundial. Sus distinguidos servicios le valieron convertirse en el militar más joven en recibir la orden Pour le Mérite, la más alta condecoración otorgada por el Ejército alemán.

Durante los años de la República de Weimar, Jünger continuó publicando sus experiencias de guerra y también contribuyó en revistas y periódicos con artículos sobre temas políticos. Participó en círculos nacionalistas y militaristas sin llegar a unirse al Partido Nazi ni a brindarle apoyo. (Más adelante, a partir de la Segunda Guerra Mundial, Jünger renunció a la filiación conservadora de su juventud y adoptó una postura más pacifista.) En la época de la república, Jünger también publicó narrativa, como las dos versiones de El corazón aventurero (1929 y 1938), así como penetrantes trabajos de crítica sociocultural de la sociedad moderna urbana e industrial, entre los cuales destaca como el ejemplo más logrado el tratado El trabajador (1932).

Lo escrito por Jünger durante los años de Weimar sobresale entre las obras más representativas de este periodo de la historia alemana y de la sociedad moderna en su totalidad, en tanto que aborda aspectos clave de las culturas europeas y no europeas de su tiempo, como el impacto de la guerra y la tecnología sobre la cultura y las percepciones de los individuos.

\section{El contexto histórico: la guerra, Weimar y la crisis}

Debido al despliegue sin precedentes de los recursos de la movilización de masas y la guerra industrial, y debido a los mundos y a las concepciones del mundo que destruyó y que ayudó a dar a la luz, en Alemania la Primera Guerra Mundial fue experimentada primordialmente como una ruptura sin paralelo de la continuidad histórica. Se percibió a la guerra como una gran cesura que demolió la fe optimista en la narrativa del progreso y trajo una atmósfera de insistente pesimismo en el cual prosperó la idea de "crisis". ${ }^{1}$ Un libro muy influyente, La decadencia de Occidente, publicado por Oswald Spengler en 1918, encarnó intelectualmente la disposición moral y espiritual de los tiempos. Los primeros años de la República de Weimar, una intensa sucesión de revueltas, guerras intestinas, asesinatos políticos, golpes de Estado

${ }^{1}$ Martin Jay, Songs of experience: Modern American and European variations on a universal theme, 2006, Berkeley, University of California Press, p. 314, y Charles Bambach, "Weimar philosophy and the crisis of historical thinking”, en Peter R. Gordon y John P. McCormick (comps.), Weimar thought: A contested legacy, 2013, Princeton y Oxford, Princeton University Press, p. 133. 
fallidos, ocupación extranjera, hiperinflación y descontento generalizado, únicamente consiguieron confirmar esta sensación "casi ininterrumpida" de catástrofe. No debe sorprender que la conciencia histórica de la época haya quedado marcada por una intenso sentimiento de vivir días de crisis.

El interés por la discontinuidad temporal expresado en el sentimiento de crisis no se limitaba al espacio de la conciencia histórica colectiva. Algunos de los observadores más perceptivos de la época señalaron que esta sensación de ser ajeno también afectaba el territorio de la subjetividad individual, en la que cobraba forma como ruptura de la estabilidad de la percepción. Entre estos observadores se encontraba Walter Benjamin, quien en sus escritos de los primeros tiempos de Weimar diagnosticó que la guerra causaba una crisis profunda en la noción misma de experiencia (Erfahrung). ${ }^{3}$ La vida en el

${ }^{2}$ Peter Gay, Weimar culture: The outsider as insider, 2001, Nueva York, Norton and Company, p. 11.

3 "Never has experience been contradicted more thoroughly: strategic experience has been contravened by positional warfare; economic experience, by the inflation; physical experience, by hunger; moral experiences, by the ruling powers. A generation that had gone to school in horse-drawn streetcars now stood in the open air, amid a landscape in which nothing was the same except the clouds and, at its center, in a force field of destructive torrents and explosions, the tiny, fragile human body." Walter Benjamin, "Experience and Poverty", en Selected Writings, vol. 2, 1999, Cambridge y Londres, The frente, al imponer agotadoras exigencias sobre los sentidos de los ejércitos, había dejado un vacío en mentes y cuerpos que hizo irrumpir una sensación de urgencia radical. De manera más específica, la guerra expuso a los soldados a una sensación más intensa de que el presente estaba dislocado del pasado y del futuro. Así, la experiencia del tiempo en el campo de batalla se caracterizó por el aislamiento extremo del presente con respecto al fluir del tiempo. Se trató de una experiencia que significaba una especie de refutación tangible de proposiciones filosóficas (como las de Husserl o Bergson) que consideraban que el tiempo es un flujo continuo e ininterrumpido. ${ }^{4}$ Es una de las razones por las que el instante como categoría temporal se colocó en el centro de la conciencia histórica durante el periodo de entreguerras.

\section{Instante, crisis y modernidad}

Dadas las características definitorias del periodo, incluso se podría hablar de la existencia de una "afinidad electiva" entre las experiencias de crisis y ruptura en la continuidad histórica que conllevan, por un lado,

Belknap Press of Harvard University Press, trad. al inglés de Rodney Livingston, p. 732.

${ }^{4}$ Stephen Kern, The culture of time and space: 1880-1918, 1983, Cambridge, Massachusetts y Londres, Harvard University Press, pp. 293-294. 
los acontecimientos de Weimar y la guerra, y, por el otro, la noción de instante como artilugio intelectual (el momento presente, el ahora aislado de discontinuidad abrupta). El sustento para esta afinidad reside en el hecho de que el instante suscita ciertas cuestiones teóricas que permanecerían en la oscuridad debajo de una visión de temporalidad anclada en la duración y el flujo. Por ejemplo, debido a su vínculo con lo repentino, el instante implica una sensibilidad única, en armonía con lo excepcional y lo inesperado, y ello es fundamental para inteligir la naturaleza de la percepción y la conciencia históricas en Europa durante las primeras décadas del siglo XX. Al elaborar sobre esta característica distintiva del instante, es posible identificar una comunidad de significados entre los conceptos de crisis, instante y modernidad. Como afirma Reinhart Koselleck, la modernidad (Neuzeit) está basada sobre dos determinantes de temporalidad, aceleración y otredad del futuro..$^{5}$ En consecuencia, la experiencia moderna de la temporalidad

5"Two specific temporal determinants characterize the new experience of transition: the expected otherness of the future and, associated with it, the alteration in the rhythm of temporal experience: acceleration, by means of which one's one time is distinguished from what went before." Reinhart Koselleck, "Neuzeit': Remarks on the semantics of modern concepts of movement", en Futures past: On the semantics of historical time, 2004, Nueva York, Columbia University Press, p. 241. se percibe con una sensación recurrente de sorpresa ante un futuro desconocido que siempre se está actualizando. El instante se presenta a sí mismo como la categoría natural para encuadrar estos encuentros acelerados con lo inesperado y lo nuevo, los cuales caracterizan a la modernidad.

Desde la perspectiva de la comunidad de significados, se podría argumentar que alrededor de los años de la Gran Guerra, el instante se convirtió en el motivo unificador de un nuevo "régime d'historicité" específicamente moderno, esto es, en aquello que el historiador francés François Hartog entiende como el modo particular en que un agente histórico se relaciona con la temporalidad histórica. ${ }^{6} \mathrm{Al}$ parecer, Oswald Spengler avanzaba en esta dirección cuando escribió en $L a$ decadencia de Occidente:

En el mundo clásico los años del mundo no desempeñaban ninguna función, en el mundo de la India importaban muy poco; pero aquí tienen importancia la hora, el minuto, incluso el segundo. Ni un griego ni un indio pudieran haber tenido idea de la trágica tensión de una crisis histórica como la de agosto de 1914, cuando hasta los momentos parecían de importancia sobrecogedora. ${ }^{7}$

${ }^{6}$ François Hartog, Régimes d'historicité: Présentisme et expériences du temps, 2003, París, Seuil, p. 19.

${ }^{7}$ Der Untergang des Abendlandes, I, p. 176, citado por Stephen Kern, op. cit., p. 258. 


\section{Jünger y la experiencia}

¿Cómo acomodar la obra literaria de Ernst Jünger en este marco? Quisiera argumentar que los escritos del periodo de entreguerras de Jünger se destacan como una de las ilustraciones literarias más evocativas de la afinidad antes mencionada entre las experiencias de ruptura y crisis y el instante como categoría temporal. Las obras más características de este periodo de Jünger (los diarios de guerra, la narrativa y los poemas en prosa, así como sus ensayos sobre temas sociales y políticos) proporcionan un vívido retrato de la sensación de crisis prevaleciente de la época. En estos escritos, Jünger explora la inmediatez de la experiencia vivida y alcanza un resultado importante: la formulación de una contraparte subjetiva e individual a la noción de la ruptura histórica.

La exploración de Jünger debe situarse en un contexto más amplio, el de las discusiones acerca de la naturaleza de la experiencia y la búsqueda de nuevas clases de experiencias en el pensamiento y la literatura del siglo XXI. Este contexto cuenta la historia de los esfuerzos de la cultura moderna por enfrentar la cuestión de la posibilidad y, en términos de una relación existencial con el tiempo abstracto de la modernidad, el "tiempo abstracto y homogéneo" que resulta al generalizar el tiempo abstracto del capitalismo, la tecnología y la ciencia. ${ }^{8}$ La mayor parte de las respuestas que se han dado (las filosofías de Bergson y Heidegger, por nombrar dos) compartieron una ambición común, recuperar la experiencia vivida.

\section{La guerra y la experiencia concreta: La guerra como experiencia interior}

En particular, la experiencia vivida aparece en un ensayo de Jünger escrito en 1922, La guerra como experiencia interior (Der Kampf als inneres Erlebnis), apasionada y controvertida meditación acerca del significado espiritual de las acciones de guerra. Acaso la característica más notable del ensayo sea la celebración del combate como una experiencia concreta, una auténtica Erlebnis, la palpable respuesta intuitiva anterior a toda abstracción intelectual. ${ }^{9}$ El culto que Jünger rinde a la Erlebnis es el vitalismo de la acción valorada en sí, una representación del encuentro con el peligro como la manera más alta posible de vivir una vida intensa. Jünger escribe: "El espíritu del guerrero, el

${ }^{8}$ Charles Taylor, Sources of the self: The making of the modern identity, 1989, Cambridge y Londres, Harvard University Press, p. 288.

${ }^{9}$ Martin Jay, "Experience without a subject", en Cultural semantics: Keyword of our time, 1998, Amherst, University of Massachusetts Press, p. 48. 
exponerse al riesgo incluso por la más mínima idea, pesa más en la balanza que cualquier reflexión acerca del bien y del mal [...] Queremos mostrar lo que tenemos; así, si caemos, habremos vivido con total plenitud". ${ }^{10}$

\section{La guerra y la experiencia de lo repentino: \\ Tempestad de acero}

El estilo literario de Jünger se caracteriza, sobre todo, por un énfasis vehemente sobre lo que el autor considera el atributo más sobresaliente de la experiencia en la guerra: el elemento de lo repentino. Con la publicación en 1920 de Tempestad de acero (Im Stahlgewittern), las memorias de su tiempo en las trincheras, Jünger sobresale como testigo agudamente perceptivo de lo repentino, que se convertía en una de las categorías centrales de la percepción en la modernidad. Con esta obra, Jünger parece afirmar que la ruptura de la continuidad histórica epitomizada por la guerra se reflejaba de manera sistemática en el terreno de la subjetividad, de modo por demás evidente en el quiebre paralelo sobre la continuidad de la percepción: el advenimiento y proliferación de choques y experiencias de carácter abrupto.

${ }^{10}$ Ernst Jünger, "Fire”, en Anton Kaes, et al., The Weimar Republic Sourcebook, 1994, los Ángeles, University of California Press, p. 20.
En el campo de batalla, un soldado vive bajo la amenaza permanente de un fallecimiento inesperado y aprende a establecer una asociación automática entre la interrupción de la regularidad y la posibilidad de la muerte. En las memorias de Jünger, la Fronterlebnis llega a convertirse en un objeto de atención detallada y metódica: la irrupción de lo repentino es narrada de manera dinámica y se transforma en el tema principal de la experiencia sensible durante la guerra. Más aún, Jünger disecta los "decisivos momentos asesinos" ${ }^{\prime 11}$ de la batalla, cuando la experiencia del peligro se presenta en forma de una "iluminación profana": los momentos cuando el relámpago del peligro implica un significado mayor, pues asume el propósito de una revelación. En un segundo, lo excepcional revela un aspecto de la verdad que, si no fuera por el instante de peligro, quedaría oculto casi por completo tras el velo de la normalidad promedio.

\section{Terror y fenomenología de lo repentino: \\ El corazón aventurero}

La fascinación que Jünger sentía por lo repentino en el campo de batalla lo orilló a acuñar una categoría especí-

${ }^{11}$ Ernst Jünger, Storm of Steel, 2004, Nueva York, Penguin Classics, p. 33. 
fica para este tipo de percepción: la noción de terror (Schreken). En El corazón aventurero, volumen de narraciones breves y poemas en prosa de 1929 (substancialmente corregido y publicado como nueva edición en 1938), Jünger refina en lo filosófico su aproximación a la temporalidad instantánea desde el punto de vista privilegiado de esta novedosa perspectiva. Las epifanías del campo de batalla mencionadas con anterioridad se vuelven materia prima para que Jünger forme y estructure una estética del terror concebida en torno a los conceptos de conmoción y sorpresa. ${ }^{12}$ Un ejemplo representativo: la escena primigenia de la "impresión demoniaca", cuando algo que se creía que estaba muerto vuelve a la vida o viceversa. Es el instante terrorífico "cuando lo vivo y lo muerto intercambian sus lugares", ${ }^{13}$ el momento que rehúye toda explicación racional.

Como principio estructural del terror es igualmente importante aquello que Jünger denomina "el otro" (das Andere), término que se refiere al momento crítico en que dos fenómenos diferentes colisionan y desencadenan la irrupción de lo inesperado, una como puerta de entrada al reino de lo demoniaco. Cuando "lo otro"

\footnotetext{
${ }^{12}$ Karl-Heinz Bohrer, Die Ästhetik des Schreckens: Die pessimistische Romantik und Ernst Jüngers Frühwerk, 1978, Munich, Carl Hanser Verlag, p. 187.

${ }^{13}$ Ibid., pp. 158-159.
}

aparece (por ejemplo, cerca de la muerte), uno siente como si el suelo que pisa desapareciera súbitamente; una sensación de vacío absoluto atenaza a la subjetividad, como si uno cayera por una zanja abierta. ${ }^{14}$

De hecho, construida sobre esta tipología del terror, en El corazón aventurero Jünger esboza lo que pudiera denominarse la fenomenología de lo repentino, una descripción meticulosa del contenido de la conciencia al momento de encontrarse con acontecimientos inesperados. ${ }^{15}$ Por ejemplo, Jünger proporciona una definición fenomenológica del terror desde la perspectiva de la temporalidad instantánea: el terror es aquello que "se extiende entre haber reconocido la caída y la caída misma". ${ }^{16}$ Esto queda ilustrado de forma paradigmática por la experiencia de Jünger cuando fue herido en la guerra, ocasión en la cual la "mayor de las revelaciones" ocurrió en "un momento inconcebiblemente breve": ${ }^{17}$ mientras yacía herido sobre el suelo, Jünger sufrió una transición abrupta desde

${ }^{14}$ Bohrer subraya la importancia de la metáfora de "la caída" de Jünger, "símbolo de la época" en tanto que es la imagen que representa la totalidad de la teoría de la percepción estética de Jünger; véase Bohrer, op. cit., p. 186.

${ }^{15}$ Ernst Jünger, The adventurous heart: Figures and capriccios, 2012, Candor, Telos Press, trad. al inglés de Thomas Friese.

${ }^{16}$ Ibid., p. 7.

${ }^{17}$ Ibid., p. 132. 
un estado de voluntarismo impulsivo hasta una actitud de contemplación intensa. Fue como "si hubiera existido, dentro del espacio del campo de batalla, un espacio más profundo, una cámara más secreta". Jünger escribe que en esos momentos "se desgarran las costuras del mundo" y "aparecen las fisuras que nos permiten adivinar los secretos arquitectónicos que suelen estar ocultos para nosotros". ${ }^{18}$

Jünger incluso adopta una postura filosófica para inteligir estos relámpagos de sabiduría instantánea, estas fracturas en el tejido de la percepción ordinaria: "escepticismo intuitivo" (Anschaulicher Skeptizismus). La frase designa "aquella forma de intuición muy lejana de la norma" 19 suscitada por estados de excepción perceptual como el dolor y el peligro, estados en que "se levanta el velo tenue que casi siempre envuelve al mundo". ${ }^{20}$ Es posible concebir El corazón aventurero como el esfuerzo de Jünger por descifrar el significado, en términos espirituales y perceptuales, de Fronterlebnis. Es como si Jünger hubiera declarado que la experiencia radical de lo repentino, impuesta por la guerra sobre la sensibilidad individual, tuviera implicaciones filosóficas genuinas que agudizarían la comprensión del funcionamiento de la mente.

\footnotetext{
${ }^{18}$ Ibid., p. 163.

${ }^{19}$ Ibid., p. 61.

${ }^{20}$ Ibid., pp. 61-62.
}

\section{La movilización total como fuente de nuevos peligros. Movilización total, Sobre el peligro, El trabajador, Sobre el dolor}

Por más que Jünger haya insistido acerca de la naturaleza excepcional de lo repentino y lo peligroso, resulta notable que haya afirmado como una de sus ideas principales que, en las condiciones de la sociedad tecnológica de masas, tales momentos de excepción realmente constituían la nueva norma de la experiencia cotidiana. Esta afirmación es un argumento central de los escritos políticos de Jünger en esa época; notablemente en su ensayo Sobre el peligro (1931) y en la trilogía sobre la movilización de las masas, compuesta por Movilización total (1930), El trabajador (1932) y Sobre el dolor (1934). En la interpretación de Jünger, la guerra había dado a luz un mundo nuevo definido por el fenómeno de la "movilización total", esto es, el despliegue generalizado de la totalidad de los recursos de una sociedad con fines de guerra. Este proceso acaba por abarcarlo todo de manera que borra las diferencias entre combatientes y civiles, y entre el estado de guerra como situación excepcional y la vida cotidiana en tiempos de paz. En una colectividad completamente movilizada, cada ser humano se convierte en 
"obrero", es decir, en la pieza de una máquina que nunca descansa.

La movilización total inaugura una situación social marcada por la crisis permanente, por el desarreglo constante de las condiciones para la percepción y la existencia. Por tanto, el atributo que define a esta época es la posibilidad de un peligro universalmente presente. Jünger piensa que la movilización es la fuente de nuevas amenazas, de riesgos todavía más temibles que los suscitados antaño por el mundo natural. Por la movilización, la letalidad en el destino irrumpe en cada aspecto de la vida y de la sociedad, restaurando la tosca primacía de lo irracional sobre la razón. ${ }^{21}$ Así, de acuerdo con Jünger, modernidad y "progreso" —al revés que en los supuestos burgueses - engendran más, no menos, conflicto, guerra y dolor. ${ }^{22}$ Este proceso confirma que el sacrificio y el riesgo son hechos irreductibles de la vida y que la seguridad burguesa no es más que una ilusión decimonónica.

Resulta significativo que, con estos escritos, Jünger haya alcanzado un logro conceptual equivalente a sistematizar la Fronterlebnis en forma de una visión general del Estado y la sociedad. La conclusión es que

\footnotetext{
${ }^{21}$ Ernst Jünger, “On Danger”, en Kaes et al., op. cit., pp. 369-373.

${ }^{22}$ Ernst Jünger, On Pain, 2008, Candor, Nueva York, Telos Press, trad. al inglés de David C. Durst.
}

las condiciones sensoriales del soldado en el frente de batalla no eran, después de todo, una excepción, sino que verdaderamente constituían el primer paso en la transformación general de la naturaleza de la experiencia.

\section{Conclusión: implicaciones filosóficas}

Los escritos de entreguerras de Jünger ilustran poderosamente el ámbito teorético del instante en tanto que dimensión perceptual y categoría de análisis. De esta afirmación se desprenden dos importantes implicaciones filosóficas. La primera es que lo escrito por Jünger en tiempos de Weimar ayudan a comprender que el "régimen de historicidad" del instante funciona sobre la base propiamente histórica de la experiencia colectiva, así como en el dominio individual de la percepción subjetiva. La temporalidad instantánea de la crisis y la ruptura repentina, tal como las presenta Jünger, se convierten en una categoría que postula de manera simultánea dos importantes dimensiones del tiempo: el elemento individual de la subjetividad y el aspecto colectivo de la historicidad. El instante es una especie de "bisagra que, sin desdibujar las diferencias entre ambos, los articula en un solo marco de 
experiencia". La segunda es que los ensayos, memorias y narraciones de Jünger contribuyen a la comprensión de que el instante es, para decirlo en los términos de Reinhart Koselleck, un concepto que al mismo tiempo registra y genera la experiencia. ${ }^{23}$ Como lo confirman los escritos de Jünger sobre la guerra, por un lado el instante es una categoría apropiada para registrar las crisis y la ruptura histórica. Por el otro, como lo muestran las ideas de Jünger acerca del horror y el peligro, el instante, una vez que ha sido codificado como figura de la percepción, se transforma en una estructura que permite generar nuevas experiencias. En cualquiera de sus numerosas encarnaciones, el instante de crisis de Jünger se revela como la manifestación arquetípica de la temporalidad repentina, ese concepto clave que expande el espacio de la conciencia y la acción en la modernidad.

${ }^{23}$ Koselleck, op. cit., p. 272. 
CITAM Derechos Reservados.

La reproducción total o parcial de este artículo se podrá hacer si el ITAM otorga la autorización previamente por escrito. 